\title{
Sarcopenia in Cancer Patients
}

\section{Andree Kurniawan}

Department of Internal Medicine, Faculty of Medicine, Pelita Harapan University, Tangerang, Indonesia

\section{ARTICLE INFO}

Received : 24 June 2019

Reviewed : 28 June 2019

Accepted : 27 September 2019

\section{Keywords:}

cancer, muscle mass, muscle strength, physical performance, sarcopenia
*Corresponding author:

Department of Internal Medicine, Faculty of Medicine, Pelita Harapan University, Tangerang, Indonesia A125ee@yahoo.com

\begin{abstract}
A BSTRACT
Introduction: Sarcopenia in cancer patients, especially in advanced stage, recently known as an emerging problem. Firstly, sarcopenia is found in elderly patients. The diagnosis of sarcopenia needs evaluation of muscle composition and function and physical activity. Sarcopenia will give negative impacts such as increased mortality, chemo-toxicity, and decreased quality of life. Here, we review the current evidence describing the definition, impact, risk factors, mechanisms, diagnosis and treatment of sarcopenia in cancer patients.
\end{abstract}

Method: We identified 48 studies and/or review articles evaluating sarcopenia in cancer patients by searching PubMed and EMBASE databases.

Results: Sarcopenia is reported across all stages and types of cancers. There is a new definition of sarcopenia that is reported in 2019 paper. The risk factors or causes of sarcopenia in cancer are complex depending on the clinical settings of each patient. SARC-F questionnaire can be used to screen cancer patients in clinical settings. The diagnostic evaluation and cut-off measurement of sarcopenia especially in cancer varied across studies. The loss of muscle mass that happens during chemotherapy will make a poor prognosis. Sarcopenia can worsen chemotherapy toxicity. Combination exercise with adequate dietary supplementation, adequate energy, and protein are important in the management of sarcopenia in cancer patients.

Conclusions: Patients with cancer belong to a population at risk of developing sarcopenia before and after chemotherapy. Sarcopenia diagnosis needs the evaluation of muscle mass and muscle strength or physical performance. Physical activity exercise is the best strategy to reduce sarcopenia in cancer patients.

\section{INTRODUCTION}

Sarcopenia is defined as decreasing muscle mass and function. Sarcopenia is commonly found in malnourished and cachexia cancer patients [1,2]. The prevalence of sarcopenia varied from $13 \%$ to $61 \%$ in different cancer types. Prevalence of sarcopenia in cancer varied depending on types, stadium, location of cancer, measurement method, cut-off, and definition of sarcopenia. Late-stage cancer and gastrointestinal cancer had higher prevalence of sarcopenia [3].

Decreasing muscle mass did not exactly correlate with muscle strength [4,5]. Manini and Clark [6] suggest the definition of dynapenia to describe loss of muscle strength. The diagnosis of sarcopenia needs evaluation of muscle mass and strength together with physical performance. Several modalities had been used to evaluate those parameters [7].

Sarcopenia has an unfavorable impact on cancer. The sarcopenia in cancer will increase mortality risk from 1.11 to 4.84 times compared to those without sarcopenia [8,9]. Sarcopenia also decreased response to chemotherapy and cancer survival. In the end, it will decrease the quality of life of patients with cancer [3]. In this literature review, we will evaluate current articles related to sarcopenia and cancer.

\section{Search Strategies}

A comprehensive search of literature was conducted in the PubMed (NIH) and EMBASE databases (January 1989 to January 2019) using keyword combinations of the medical subject headings (MeSH) of 'sarcopenia', 'muscle mass', 'muscle strength', 'physical performance', 'exercise', 'cancer', and 'neoplasm'. Relevant reference lists were also manually searched. Studies on childhood cancer and hematological diseases were excluded.

\section{Definition of Sarcopenia}

Sarcopenia is defined as a deprivation of muscle mass and function. In 1988, Rosenborg for the first time used the concept of sarcopenia as progressive loss of skeletal muscle mass in elderly. Decreasing of lean body mass will impact decreasing physical performances which are correlated with increasing age [1,2]. Firstly, Baumgartner used the term "sarcopenia" to describe 
decreasing muscle mass because of age. Sarcopenia in Greek term means poor of flesh [7].

Based on the European Working Group on Sarcopenia in Older People (EWGSOP), sarcopenia is a syndrome characterized by decreasing muscle mass and function with impact on physical disability, and poor quality of life and survival. Based on those criteria, sarcopenia is diagnosed if there is a decreasing of muscle mass and function [10]. Diagnosis criteria of sarcopenia need low muscle mass and either low muscle strength or disturbance of physical performances in daily life. Muscle strength is not correlated solely on muscle mass. Those relationships were not linear $[10,11]$. As a result, the diagnosis of sarcopenia needs evaluation of muscle mass and strength together with physical performances as can be seen in Table 1 .

Sarcopenia can be divided into 3 stages. If low muscle mass occurs without any sign of low muscle strength and physical performance, the condition is defined as pre-sarcopenia. This stage could be identified with good technique to measure muscle mass exactly based on the normal value in the population. Sarcopenia was defined if there is low muscle mass accompanied by either low muscle strength or physical performance. If low muscle mass, low muscle strength, and low physical performance were found, the condition is categorized as severe sarcopenia [10].

In 2018, EWGSOP2 uses low muscle strength as the primary parameter of sarcopenia; muscle strength is currently the most reliable measurement of muscle function. Sarcopenia is probable when low muscle strength is detected. Sarcopenia diagnosis is confirmed by the presence of low muscle quantity or quality [12]. The 2018 operational definition of sarcopenia can be seen in Table 2.

\section{Mechanisms of Sarcopenia in Cancer}

There are several proposed mechanisms of sarcopenia. Primary, age-related, sarcopenia is associated with sex hormones, apoptosis, and mitochondrial dysfunction, anorexia of aging. Secondary sarcopenia starts from the mechanism involving neurodegenerative processes such as motor neuron diseases and endocrinopathy which is associated with a reduction of anabolic hormone levels (such as growth hormone, insulin-like growth factor-1 (IGF-1), testosterone, vitamin $D$, increased cortisol, abnormal thyroid function, insulin resistance), pro-inflammatory cytokines (such as interleukin-1, interleukin-6 and tumor necrosis factoralpha), and inadequate nutrition or malabsorption and disuse situations (such as the immobilization syndrome, physical inactivity) [10,13-15].

There are several mechanisms that might contribute to the onset and progression of sarcopenia. Sarcopenia can be divided based on the causes of primary sarcopenia or age-related sarcopenia and secondary sarcopenia. Several factors, such as sex hormones, apoptosis, and mitochondrial dysfunction, may have roles in the development of sarcopenia. Secondary sarcopenia could be divided into activity-related sarcopenia, disease-related sarcopenia, and nutrition-related sarcopenia. Activityrelated sarcopenia can result from bed rest, sedentary lifestyle, deconditioning or zero-gravity conditions. Disease-related sarcopenia is associated with advanced organ failures such as heart, lung, liver, kidney, or brain failure, or inflammatory diseases (cancer or endocrine disease). Lastly, nutrition-related sarcopenia is resulted from inadequate dietary intake of energy and/or protein, for instance in conditions of malnutrition and gastrointestinal disorders [10]. The summary of mechanisms of sarcopenia can be seen in Figure 1 .

Table 1. Diagnosis criteria of sarcopenia [10]

Diagnosis based on criteria 1 AND criteria 2 or 3

1. Low muscle mass

2. Low muscle strength

3. Low physical performances.

Table 2. Operational definition of sarcopenia (2018) [12]

Probable sarcopenia is identified by Criterion 1.

Diagnosis is confirmed by additional documentation of Criterion 2.

If Criteria 1,2 , and 3 are all met, sarcopenia is considered severe.

Low muscle strength

Low muscle quantity and quality

Low Physical performance

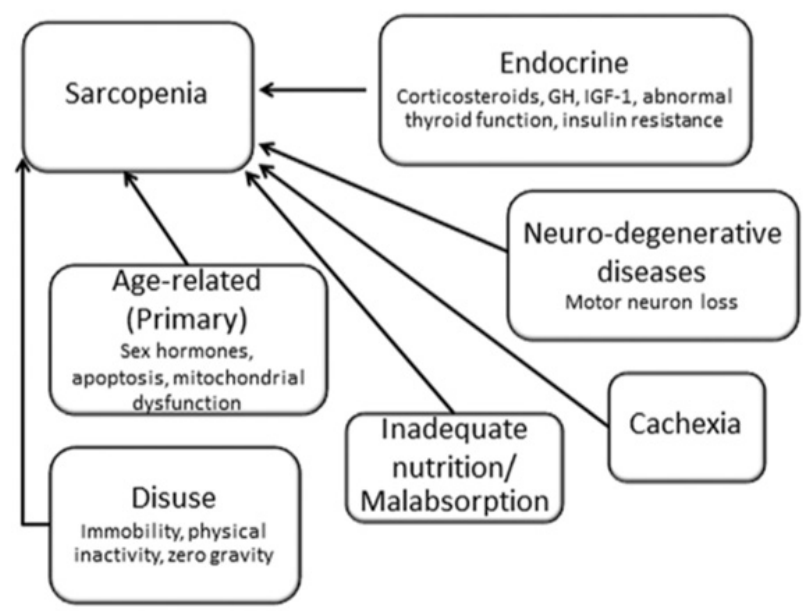

Figure 1. Mechanisms of sarcopenia [10].

\section{Impact of Sarcopenia in Cancer}

Sarcopenia in cancer patients will develop higher chemotherapy toxicity because of oncology therapy, as a result of decreased tolerance to chemotherapy, decreased 
chemotherapy doses and delayed chemotherapy schedule. Toxicity increment caused by those risks varied from 1.6 to 13 times depends on the type of chemotherapy drugs given [3]. The possible explanation for this chemotherapy toxicity is because chemotherapy dose was defined based on the calculation of body surface area consist of height and weight to predict the soft tissues.

Sarcopenia patients will get greater toxicity after oncology therapy compared to those without sarcopenia, due to decreased tolerance to therapy by decreased chemotherapy dose or delayed chemotherapy schedule. The risk of toxicity varied between 1.6 to 13 times depends on the type of chemotherapy drugs given [3]. The possible explanation of chemotherapy toxicity in sarcopenia is because the chemotherapy drug dose evaluation is based on body surface area comprise of height and weight to predict body tissues. It did not consider adipose mass that is the biggest tissue in the body and this one is the most difficult part to predict. The body composition difference is varied between the different classes of body mass index, so the body tissue estimation using body surface is not precise $[16,17]$. Because of this difference, sarcopenia patients tend to get increased chemotherapy toxicity [18].

Prado and colleagues found that lean body mass correlated with chemotherapy distribution volume and there was small correlation between lean body mass and body surface area $\left(r^{2}=0.37\right)$, especially in obese patients. The variation can be increased to three times effective distribution volume for each chemotherapy drug given to every unit body surface area. They also explained that lean body mass and aspartate transaminase (AST) contribute to $33 \%$ variation in Epirubicin clearance [19]. This explains overestimation of posology drug when body surface area is used. This variation can be bigger concomitant with increased body mass index [20].

In cancer patients with sarcopenia whose chemotherapy doses were calculated based on the body weight, in the settings distribution volume lower will impact higher chemotherapy drug concentration in a relatively short period. In those conditions, there is difficulty in metabolizing and clearing chemotherapy drugs from systemic circulation [21].

\section{Risk Factors of Sarcopenia in Cancer Body composition}

The important factor in metabolism many drugs include chemotherapy is body composition. Patients with low lean body mass (LBM) will impact to low distribution volume of cytotoxic chemotherapy drugs. The previous study explained that patients who got chemotherapy 5-fluorouracil (5-FU) doses based on body weight associated with increased incidence overall toxicity. Another study done by Gusella et al. [22] showed that LBM and total body water (TBW) were the better prediction pharmacokinetic clearance and distribution volume than body surface area (BSA) or bodyweight.

Aslani et al. [23] study done in 31 patients who got cyclophosphamide, methotrexate, and 5-FU with calculated doses with LPB, there were increased 28 times relative risk neutropenia grades 3 and 4 in patients with LBM less than $89 \%$ than normal after adjusted with age and sex. This study also showed that chemotherapy toxicity correlated inverse with muscle mass.

\section{Age}

The incidence of cancer increased with age and more than $50 \%$ of all cancer diagnosed after 65 years old [24]. Decreased muscle mass associated with age started after the $5^{\text {th }}$ decade and those decreased was $1.9 \mathrm{~kg}$ every decade in males and $1.1 \mathrm{~kg}$ every decade in female. The loss especially types 2 muscle spindles because of progressive loss of denervation and motor neuron. Comorbidity such as type 2 diabetes and ischemic stroke had a role through inflammation pathway, disturbed metabolism and deconditioning $[25,26]$. Because the muscle loss correlated with age, chemotherapy drugs are given in elderly increased toxicity risk.

\section{Malnutrition}

Cancer patients with severe malnutrition will develop cachexia. From a survey of 1453 oncology outpatient clinics, there were reported $32 \%$ increased risk of malnutrition [27]. In condition of negative energy balance, amino acids from muscle protein will be used as energy sources and will impact decreased muscle protein [28]. Decreasing total protein muscle will contribute to decreased muscle function. In study of colorectal cancer with malnutrition, there is decreasing $29 \%$ handgrip strength and $12.2 \mathrm{~kg}$ total body muscle when compared to them without malnutrition [29].

\section{Immobilization}

Patients with cancer on some occasions have decreased physical daily activity. In post-operation period, they usually stayed in prolonged bedridden that will impact decreased muscle mass similarly found in elderly patients. Immobilization more than 10 days will decrease isokinetic muscle strength and decreased $1.5 \mathrm{~kg}$ total body muscle mass especially in lower extremities [30].

\section{Inflammation}

Cancer is chronic inflammation. A study in mice with cancer showed increased in pro-inflammation cytokines such as TNF-alpha, I1a-beta and interferon-gamma. This proinflammation cytokines activated nuclear factor kappa beta signal in muscle and then started transcription ligase ubiquitin, atropine-1, and Murf1. The end product was 
activated this pathway and developed muscle protein degradation [31]. Those activated pathways also reported in newly diagnosed cancer patients. Those phenomena supported that inflammation response to cancer cells directly start with first phase muscle degradation and this one had happened in early-stage until developed decreasing of muscle mass detected clinically [32].

\section{Cancer cell metabolism}

The cancer cell is active metabolism through glycolysis cycle that produced prime energy. The cancer cell will take a sum of glucose by competing from the host cell. A recent study showed that cancer cell depends on amino acid glutamine [33,34]. As a result, cancer patients had negative protein balance, will develop malnutrition and at last decreased muscle mass.

\section{Malnutrition-associated Sarcopenia}

The sarcopenia phenotype is also associated with malnutrition, regardless of if the malnourished condition because of low dietary intake (starvation, inability to eat), reduced nutrient bioavailability (diarrhea, vomiting) or high nutrient bioavailability (inflammatory disease such as cancer). Low muscle mass has recently been considered as part of definition of malnutrition. Also in malnutrition, low-fat mass is usually present but is not necessarily the case in sarcopenia $[35,36]$.

\section{Screening of Sarcopenia}

Variety tests and tools are now available for screening sarcopenia in practice and in research. In clinical practice, case finding may start when patients report symptoms and signs of sarcopenia. Symptoms like falling, feeling weak, slow walking speed, difficulty rising from a chair or weight loss/muscle wasting should be considered having sarcopenia. In such cases, further testing and evaluation of sarcopenia are recommended [37].

European Working Group Sarcopenia in Older People (EWGSOP) recommends the use of the SARC-F questionnaire to evaluate self-reports from patients with signs of sarcopenia. SARC-F can be readily used in community healthcare and other clinical settings. The
SARC- $F$ is a 5-item questionnaire that is self-reported by patients as screen for sarcopenia risk [38]. Responses are based on the patients' perception of their limitation in strength, walking ability, rising from a chair stair climbing and experiences of falls. The tools have been validated in larger population especially in Asian population in a study of Chinese men and women [39].

SARC-F has low to moderate sensitivity and very high specificity to predict low muscle strength. So, SARC-F will likely to detect severe cases. SARC-F is an inexpensive and convenient method for sarcopenia risk screening [40]. Alternatively, clinicians may use a formal case-finding instrument such as using the Ishii screening test, a method that estimates the probability of sarcopenia using an equation-derived score based on three variables-age, grip strength and calf circumference [41].

\section{Diagnosis of Sarcopenia}

Based on the definition of sarcopenia comprise of muscle mass and strength, to diagnosis need examination both of them. Muscle mass evaluation is done from examination body composition using dual-energy X-ray absorptiometry (DXA) and bioelectrical impedance (BIA). Muscle mass examination also can be done through cross-sectional area evaluation that correlated with body composition. A tool to evaluate this one can use computerized tomography (CT), magnetic resonance imaging (MRI) and ultrasound [42].

Function exam or muscle strength can be done with handgrip strength exam, quadriceps muscle strength and skeletal muscle index..$^{43}$ Based on recommendation of EWGSOP, low handgrip strength was $30 \mathrm{~kg}$ in male and $20 \mathrm{~kg}$ in female. Peak expiration flow can be used to evaluate respiratory muscle strength if there are no lung problems. This exam is cheap, simple, available in many places and has prognostic value $[44,45]$.

Evaluation physical performance comprises of walking speed, six-minute walking test, and walking upstairs test. Walking speed less than $0.8 \mathrm{~m} / \mathrm{s}$ are categorized as poor physical performance. To evaluate muscle cell structure, muscle biopsy can be done to evaluate muscle morphology, biochemical indicator and gene expression profile [42]. Modality to evaluate sarcopenia summarize in Table 3.

Table 3. Measurement of sarcopenia parameters $[10,12,42,46]$

\begin{tabular}{|c|c|c|c|c|c|}
\hline \multirow{2}{*}{ Measurement } & \multirow{2}{*}{ Tools } & \multicolumn{2}{|c|}{ Cut-off values AWGS } & \multicolumn{2}{|c|}{ Cut-off values EWGSOP } \\
\hline & & Male & Female & Male & Female \\
\hline \multirow{2}{*}{$\begin{array}{l}\text { Skeletal muscle } \\
\text { mass or quality }\end{array}$} & Dual energy X-ray absorptiometry (DXA) & $7 \mathrm{~kg} / \mathrm{m}^{2}$ & $5.4 \mathrm{~kg} / \mathrm{m}^{2}$ & $7 \mathrm{~kg} / \mathrm{m}^{2}$ & $6 \mathrm{~kg} / \mathrm{m}^{2}$ \\
\hline & Bio-impedance analysis (BIA) & $7 \mathrm{~kg} / \mathrm{m}^{2}$ & $5.7 \mathrm{~kg} / \mathrm{m}^{2}$ & $<10.76 \mathrm{~kg} / \mathrm{m}^{2}$ & $<6.76 \mathrm{~kg} / \mathrm{m}^{2}$ \\
\hline \multirow{2}{*}{ Muscle strength } & Handgrip strength (HS) & $26 \mathrm{~kg}$ & $18 \mathrm{~kg}$ & $27 \mathrm{~kg}$ & $16 \mathrm{~kg}$ \\
\hline & Knee flexion/extension (quadriceps strength) & $18 \mathrm{~kg}$ & $16 \mathrm{~kg}$ & - & - \\
\hline Physical performance & 6-m/4-m usual gait speed & $0.8 \mathrm{~m} / \mathrm{s}$ & $0.8 \mathrm{~m} / \mathrm{s}$ & $0.8 \mathrm{~m} / \mathrm{s}$ & $0.8 \mathrm{~m} / \mathrm{s}$ \\
\hline
\end{tabular}

AWGS: Asian working Group on Sarcopenia; EWGSOP: European Working Group on Sarcopenia. 
To screen sarcopenia based on Asian Working Group on Sarcopenia (AWGS) recommendation, start with gait speed or physical performance and handgrip strength. If one of them is abnormal, proceed with muscle mass evaluation. Sarcopenia will be diagnosed if the result of muscle mass exam below the normal cut-off. Then, if both of the examinations were normal, we can conclude there is no sarcopenia [46].

Although the cut-off of these measurements in Asians might differ from those in Caucasians. If we use the lowest $20^{\text {th }}$ percentile of gait speed in community settings according to mostly Asian studies, the cut-off would be higher than $1 \mathrm{~m} / \mathrm{s}$. Based on several epidemiological studies in Asia, the definition of low handgrip strength is $<26 \mathrm{~kg}$ for men and $<18$ $\mathrm{kg}$ for women. The AWGS recommends using height adjusted skeletal muscle mass, with suggested cut-off values of $7.0 \mathrm{~kg} / \mathrm{m}^{2}$ in men and $5.4 \mathrm{~kg} / \mathrm{m}^{2}$ in women using dual $\mathrm{x}$-ray absorptiometry (DXA). The suggested cut-off values are $7.0 \mathrm{~kg} / \mathrm{m}^{2}$ in men and $5.7 \mathrm{~kg} / \mathrm{m}^{2}$ in women when bioelectrical impedance analysis (BIA) is used. Yet, DXA is not yet available in most Asian countries because the software analysis is different from bone mineral density evaluation. BIA is an alternative inexpensive measurement for assessing muscle mass [47].

A variety of methods are being used or evaluated to determine the quantity and quality of muscle and impact of sarcopenia in a patient's QoL. These diagnostic measures are being tested for validity, reliability, and accuracy in cancer patients.

\section{Lumbar $3^{\text {rd }}$ vertebra imaging by computed tomography}

Computed tomography (CT) has been used in cancer patients to describe tumors and their response to treatment, and this technique has also been shown to give a practical and precise measure of body composition. In particular, CT images of a specific lumbar vertebral landmark (L3) are correlated significantly with wholebody muscle. This imaging method has been used to detect low muscle mass, even in patients with normal or high body weights and it also can predict prognosis. Quantification of lumbar L3 cross-sectional area also has been done by MRI $[48,49]$.

\section{Mid-thigh muscle measurement}

Mid-thigh imaging (by MRI or CT) has also been used in research studies. This is a good predictor of whole-body skeletal muscle mass and very sensitive to change. Mid-thigh muscle area is more strongly correlated with total body muscle volume than are lumbar muscle areas L1-L5 [48,49].

\section{Psoas muscle measurement}

CT-based measurement of the psoas muscle has also been reported as simple and predictive of morbidities in certain conditions (cirrhosis, colorectal surgery). Further studies need to be done to verify this method because psoas is minor muscle and it might not be the representative of overall sarcopenia $[50,51]$.

\section{Muscle quality measurement}

Muscle quality measurement is a relatively new term, referring to both micro and macroscopic changes in muscle architecture and composition and muscle function delivered per unit of muscle mass. Imaging tools such as MRI and $\mathrm{CT}$ have been used to assess muscle quality in research settings, for instance to determine infiltration of fat into muscle and use the attenuation of the muscle. Another term, muscle quality, has been applied to ratios of muscle strength to appendicular skeletal muscle mass or volume. Muscle quality has been assessed by BIA-derived phase angle measurement $[52,53]$.

\section{Treatment}

Non-pharmacological management is beneficial for sarcopenia in adults in terms of prevention and treatment such as exercise and nutrition. No Medications are currently proven to be efficacious as exercise but might be beneficial in older adults with deficiency such as testosterone, dehydroepiandrosterone (DHEA), growth hormone, and vitamin D.

\section{Exercise}

Physical activity exercise is the best strategy for primary and secondary prevention. Physical activity can change skeletal muscle directly and can repair this muscle dysfunction. Repetitive muscle contraction can stimulate physiologic changes of muscle contractile machinery, mitochondria function, metabolic settlement and intracellular signal $[42,54]$.

Physical inactivity is part of the pathogenesis of sarcopenia. Therefore, exercise can reverse or prevent the occurrence of sarcopenia. Exercise is the most effective method to improve quality of life and function in older adults [55]. Resistance exercise (RE) is the type of exercise that mainly increases muscle mass and muscle strength by directly stimulate protein synthesis especially by progressive resistance exercise (PRE). This exercise is the most commonly used in resistance therapy. It requires muscle to generate power to move or resist weight and escalate the intensity when physical capacity improves $[55,56]$. A meta-analysis and systematic review showed that progressive resistance exercise can reduce physical disability in older adults [57]. 
Aerobic exercise can benefit to improve cardiovascular fitness and endurance. It increases the cross-sectional area of muscle fibers, mitochondrial volume and enzyme activity, but it is unlikely to increase muscle hypertrophy [58]. Combinations of aerobic and resistance exercises can further improve muscle strength and function and are important to prevent and treat sarcopenia [59].

\section{Diet}

Anorexia is commonly found in cancer patients especially in the late stage. Anorexia is the commonlyused word to define reduce appetite and low intake. Inadequate protein intake is particularly the key component to develop sarcopenia. It can be the result of physiological change, comorbidities and treatmentrelated [60].

The daily recommendation of protein is about 1.5 $\mathrm{g} / \mathrm{kg} /$ day which is higher than current dietary recommendation because of increased catabolism in cancer patients. An essential amino acid (EAA) plays an important role in muscle protein synthesis and declined proteolysis. It is recommended for cancer patients to take adequate protein with a high EAA such as beef, fish, and peanuts [55].

Vitamin $D$ decreases with age as a result of an intrinsic change in vitamin D synthesis and extrinsic causes such as malnutrition and reduced sunlight exposure in elderly cancer patients and bedridden. Vitamin D deficiency is related to altered muscle morphology causing osteomalacia and myopathy, reduced muscle strength, muscle mass, and physical performances that lead to an increased risk of sarcopenia. Vitamin D supplementation does not show beneficial effects in improved muscle strength, muscle function and decreased risk of falls but co-administration with $1000 \mathrm{mg}$ of calcium in vitamin D deficient patients can decrease risk of falling. This effect is dose-dependent (700-1000 IU/day of vitamin D 3) and vitamin D level of at least $60 \mathrm{nmol} / \mathrm{L}$ is required for prevention [61-63].

There is evidence that the combination of exercise, adequate nutrition, and vitamin $D$ supplementation is superior to the exercise alone to increase muscle strength, muscle mass and gait speed in sarcopenic patients. Therefore, adequate energy and protein intake during exercise are important [60].

\section{Medications}

The mechanisms of pharmacological management of sarcopenia focus on various pathways such as androgen receptors, peroxisome proliferator-activated receptor delta, insulin-like growth factor 1, betaadrenergic receptors, neuregulins, angiotensin-converting enzymes, and inflammatory cytokines. However, no proven drug is recommended to be used in sarcopenia for prevention and treatment at present. Some medications improved muscle mass, muscle function and physical performance in animal studies but it is limited in cancer patients. Most studied drugs are testosterone, growth hormone, dehydroepiandrosterone (DHEA) and vitamin D especially in older adults [54,62].

The growth hormone is indicated in growth disorders and growth hormone deficiency. The benefits of $\mathrm{GH}$ in older adults are associated with increased total lean body mass, decreased fat mass, and decreased musclebone demineralized rate but the role in increased muscle strength, muscle power, aerobic endurance and physical performance after short treatment periods is limited. Severe side effects that are also found are joint pain, carpal tunnel syndrome, edema, carbohydrate intolerance, diabetes, and tumors [54].

DHEA is a precursor of sex hormones indicated in testosterone deficiency. It can increase bone density in older adults, and it may increase muscle strength via an increased ratio of circulating testosterone to cortisol in animal studies $[56,59]$.

\section{CONCLUSIONS}

Patients with cancer are the population at risk to develop sarcopenia before and after chemotherapy. The loss of muscle mass that happens during chemotherapy will make a poor prognosis. Sarcopenia can worse chemotherapy toxicity. Sarcopenia diagnosis needs evaluation of muscle mass and muscle strength or physical performance. Physical activity exercise is the best strategy for sarcopenia in cancer patients. The combination of adequate energy and protein with vitamin D supplementation is important.

\section{REFERENCES}

1. Rosenborg IH. Sarcopenia: origins and clinical relevance. J Nutr. 1997;127: 9905-15.

2. Rosenberg IH. Summary comments. Am J Clin Nutr. 1989;50: 1231-3.

3. Bozzetti F. Forcing the vicious circle: sarcopenia increases toxicity, decreases response to chemotherapy and worsens with chemotherapy. Annals of oncology. 2017;28: 2107-18.

4. Reed RL, Pearlmutter L, Yochum K, Meredith KE, Mooradian $A D$. The relationship between muscle mass and muscle strength in the elderly. J Am Geriatr Soc. 1991;39: 555-61.

5. Hugher VA, Frontera WR, Wood M. Longitudinal muscle strength changes in older adults: influence of muscle mass, physical activity, and health. J Gerontol A Biol Sci Med Sci. 2001;56: B209-17. 
6. Manini TM, Clark BC. Dynapenia and aging: an update. J Gerontol A Biol Sci Med Sci. 2012;67: 28-40.

7. Baumgartner RN, Koehler KM, Gallagher $D$. Epidemiology of sarcopenia among the elderly in the New Mexico. Am J Epidemiol. 1998;47(8): 755-63.

8. Harimoto N, Shirabe K, Yamashita YI. Sarcopenia as a predictor of prognosis in patients following hepatectomy for hepatocellular carcinoma. Br J Surg. 2013;100(11): 1523-30.

9. Mir O, Coriat R, Blanchet B. Sarcopenia predicts early dose limiting toxicities and pharmacokinetics of sorafenib in patients with hepatocellular carcinoma. PLoS One. 2012;7(5): e37563.

10. Cruz-Jentoft AJ, Baeyens JP, Bauer JM, Boirie Y, Cederholm T, Landi F, et al. Sarcopenia: European consensus on definition and diagnosis. Age and Ageing. 2010;39: 412-23.

11. Goodpaster BH, park SW, Harris TB. The loss of skeletal muscle strength, mass, and quality in older adults: the health, aging and body composition study. J Gerontol A Biol Sci Med Sci. 2006;61: 1059-64.

12. Cruz-jentoft AJ, Bahat G, Bauer J, Boire Y, Bauyere O, Cederholm T, et al. Sarcopenia: revised European consensus on definition and diagnosis. Age and Ageing. 2019;48: 16-31.

13. Morley JE, Anker SD, von Haehling S. Prevalence, incidence, and clinical impact of sarcopenia: facts, numbers, and epidemiology-update 2014. J Cachexia Sarcopenia Muscle. 2014;5: 253-9.

14. Kim TN, Choi KM. Sarcopenia: definition, epidemiology, and pathophysiology. J Bone Metab. 2013;20: 1-10.

15. Marcell TJ. Sarcopenia: causes, consequences, and preventions. J Gerontol A boil Sci Med Sci. 2003;58: M911-6.

16. Du Bois D, Du Bois EF. Clinical calorimetry: tenth paper. A Formula to estimate the approximate surface area if height and weight be known. Arch Intern Med (Chic). 2016;17: 863-71.

17. Morgan DJ, Bray KM. Lean body mass as a predictor of drug dosage. Implications for drug therapy. Clin Pharmacokinet. 1994;26(4): 292-307.

18. Prado CM, Baracos VE, McCargar LJ, Mourtzakis M, Mulder KE, Reiman $\mathrm{T}$, et al. Body composition as an independent determinant of 5-flurouracil-based chemotherapy toxicity. Clinical cancer research: an official journal of the American Association for cancer research. 2007;13: 3264-8.

19. Prado CM, Lima IS, Baracos VE. An exploratory study of body composition as a determinant of epirubicin pharmacokinetics and toxicity. Cancer Chemother Pharmacol. 2011;67(1): 93-101.

20. Crosby V, D'Souza C, Bristow C. Can body composition be used to optimize the dose of platinum chemotherapy in lung cancer? A feasibility study. Support care cancer. 2017;25(4): 1257-61.
21. Prado CM, Baracos VE, McCargar L. Sarcopenia as a determinant of chemotherapy toxicity and time to tumor progression in metastatic breast cancer patients receiving capecitabine treatment. Clin Cancer Res. 2009;15: 2920-6.

22. Gusella M, Tosso S, Ferrazzi E, Ferrari M, Padrini R. Relationship between body composition parameters and flurouracil pharmacokinetics. Br J Clin Pharmacol. 2002;54: 13-9.

23. Aslani A, Smith RC, Allen BJ, Pavlakis N, Levi JA. The predictive value of body protein for chemotherapyinduced toxicity. Cancer. 2000;88: 796-803.

24. Yancik R. Population aging and cancer: cross-national concern. Cancer J. 2005;11: 437-41.

25. Andersen JL. Muscle fibre type adaptation in elderly human muscle. Scand J Med Sci Sports. 2003;13: 40-7.

26. Extermann M. Measurement and impact of comorbidity in older cancer patients. Crit Rev Oncol Hematol. 2000;35: 181-300.

27. Bozzetti $F$, mariani L, Lo VS. The nutritional risk in oncology: a study of 1453 cancer outpatients. Support care cancer. 2012;20: 1919-28.

28 . Wolfe RR. The underappreciated role of muscle in health and disease. Am J Clin Nutr. 2006;84: 475-82.

29. Burden ST, Hill J, Shaffer JL. Nutritional status of preoperative colorectal cancer patients. J Hum Nutr Diet. 2010;23:402-7.

30. Kortebein P, Ferrando A, Lombeida J. Effect of 10 days of bed rest on skeletal muscle in healthy older adults. JAMA. 2007;297: 1772-4.

31. Khal J, Wyke SM, Russel ST. Expression of the ubiquitin-proteasome pathway and muscle loss in experimental cancer cachexia. Br J Cancer. 2005;93: 774-80.

32. Bossola M, Muscaritoli M, Costelli P. Increased muscle proteasome activity correlates with disease severity in gastric cancer patients. Ann Surg. 2003;237: 384-9.

33. Koppenil WH, Bounds PL, Dang CV. Otto Warburg's contribution to current concepts of cancer metabolism Nat Rev Cancer. 2011;11: 325-37.

34. Qing G, Li B, Vu A. ATF4 regulates MYC- mediated neuroblastoma cell death upon gluttamin deprivation. Cancer cell. 2012;22:531-44.

35. Cederholm T, Barazzoni R, Austin P. ESPEN guidelines on definitions and terminology of clinical nutrtion. Clin Nutr. 2017;36: 49-64.

36. Cederholm T, Jensen GI, Correia M. GLIM criteria for the diagnosis of malnutrition - A consensus report from the global clinical nutrition community. Clin Nutr. 2018; 1440.

37. Morley JE, Abbatecola AM, Argiles JM. Sarcopenia with limited mobilty: an international consensus. J Am Med Dir Assoc. 2011;12: 403-9. 
38. Malmstrom TK, Miler DK, Simonsick EM. SARC-F: a symptom score to predict persons with sarcopenia at risk for poor functional outcomes. J Cachexia Sarcopenia Muscle. 2016;7: 28-36.

39. Woo J, Leung J, Morley JE. Defining sarcopenia in terms of incident adverse outcomes. J Am Med Dir Assoc. 2015;16: 247-52.

40. Bahat G, Yilmazi O, Kilic C. Performances of SARC-F in regard to sarcopenia definitions, muscle mass and functional measures. J Nutr Health Aging. 2018 doi:10.1007/s12603-018-1067-8. Epub ahead of print.

41. Ishii S, Tanaka T, Shibasaki K. Development of a simple screening test for sarcopenia in older adults. Geriatr Gerontol Int. 2014;14(Suppl 1): 93-101.

42. Christensen JF, Jones LW, Anderson JL, Daugaard G, Rorth $\mathrm{M}$, Hojman P. Muscle dysfunction in cancer patients. Annals of oncology. 2014;25(5): 947-58.

43. Kilgour RD, Vigano A, Trutschningg B. Cancer-related fatigue: the impact of skeletal muscle mass and strength in patients with advanced cancer. J Cachexia Sarcopenia Muscle. 2010;1: 177-85.

44. Kim J, Davenport P, Sapienza C. Effect of expiratory muscle strength training on elderly cough function. Arch Gerontol Geriatr. 2009;48: 361-6.

45. Beaudart C, McCloskey E, Bruye O, Cesari M, Rolland $Y$, Rizzoli R. Sarcopenia in daily practice: assessment and management. BMC Geriatrics. 2016;170(16): 1-10.

46. Chen LK, Liu LK, Woo J, Assntachai P, Auyeung TW, Bahyah KS, et al. Sarcopenia in Asia: consensus report of the Asian working group for sarcopenia. J Am Med Dir Assoc. 2014;15: 95-101.

47. Quest editors. Growing research on sarcopenia in Asia. Geriatr Gerontol Int. 2014;14(Suppl. 1): 1-7.

48. Mortzakis M, Prado CM, Lieffers JR. A Practical and precise approach to quantification of body composition in cancer patients using computed tomography images acquired during routine care. Appl Physiol Nutr Metab. 2008;33: 997-1006.

49. Kim EY, Kim YS, Park I. Prognostic significance of CT-determined sarcopenia in patients with small-cell lung cancer. J Thocac Oncol. 2015;10: 1795-9.

50. Gu DH, Kim MY, Seo YS. Clinical usefulness of psoas muscle thickness for the diagnosis of sarcopenia in patients with liver cirrhosis. Clin Mol Hepatol. 2018;24: 319-30.
51. Baracos VE. Psoas as a sentinel muscle for sarcopenia: a flawed premise. J Cachexia Sarcopenia Muscle. 2017;8: 527-28.

52. Lynch NA, Metter EJ, Lindle RS. Muscle quality. Ageassociated differences between arm and leg muscle groups. J Appl Physiol. 1999;86: 188-94.

53. Heymsfiled SB, Gonzales MC. Skeletal muscle mass and quality: evolution of modern measurement concepts in the context of sarcopenia. Proc Nutr Soc. 2015;74: 355-66.

54. Limpawattana $\mathrm{P}$, Kotruchin P, Pongchaiyakul C. Sarcopenia in Asia. Osteoporosis and sarcopenia. 2015;1: 92-7.

55. Montero-fernandez N, Serra-rexach JA. Role of exercise on sarcopenia in the elderly. Eur J Phys Rehabil Med. 2013;49: 131-43.

56. Malafarina V, Uriz-Otano F, Iniesta R, Gil-Guerrero L. Sarcopenia in the elderly: diagnosis, physiopathology and treatment. Maturitas. 2012;71: 109-14.

57. Liu CJ, Latham N, Can progressive resistance strength training reduce physical disability in older adults? A meta-analysis study. Disabil rehabil. 2011;33: 87-97.

58. Burton LA, Sumukadas D. Optimal management of sarcopenia. Clin Interv Aging. 2010;5: 217-28.

59. Peterson MD, Rhea MR, Sen A, Gordon PM. Resistance exercise for muscular strength in older adults: a metaanalysis. Ageing Res Rev. 2010;9: 226-37.

60. Kim HK, Suzuki T, Saito K, Yoshida H, Kobayashi H, Kato $H$. effects of exercise and amino acids supplementation on body composition and physical function in community-dwelling elderly Japanese sarcopenic women: a randomized controlled trial. J Am Geriatr Soc. 2012;60: 16-23.

61. Anagnotis P, Dimopoulou C, Karras S, Lambrinoudaki I, Goulis DG. Sarcopenia is post-menopausal women: is there any role for vitamin D?. Maturutas. 2015;82(1): 56-64.

62. Daly RM. Independent and combined effect of exercise and vitamin D on muscle morphology, function and falls in the elderly. Nutrients. 2010;2: 1005-17.

63. Borst SE. Interventions for sarcopenia and muscle weakness in older people. Age Ageing. 2004;33: 548-55. 